\title{
El coip y su relación con las ciencias forenses
}

\section{The coip and its relationship with the forensic sciences}

\author{
Aurelio Agustín Quito Cortes. ${ }^{1}$ \& Benjamín Gabriel Quito Cortes. ${ }^{2}$
}

Recibido: 10-02-2019 / Revisado: 15-02-209 /Aceptado: 04-03-2019/ Publicado: 14-06-2019

\begin{abstract}
.
https://doi.org/10.32/cienciadigital.v3i1.947

The present article of forensic social expertise, has as purpose to establish the methodological process of the Penal Organic Code to follow with the own techniques of the profession and collaterals of the social sciences, The use of the same one must be considered like the practical, professional guide of the expert in the application of the forensic sciences that allows the adequate application of the format of the report of the social perica.

The application and use of it must be used under guidelines of professional ethics, transparency, objectivity and impartiality. The proper use of the same facilitates the use and management of the report format of the social expertise and projects its application to all the skills requested by the competent authority, at the national level.
\end{abstract}

Keywords: Coip, application, science, forensics

\section{Resumen.}

El presente artículo de pericia social forense, tiene como propósito establecer el proceso metodológico del Código Orgánico Penal a seguir con las técnicas propias de la profesión y colaterales de las ciencias sociales, El uso del mismo debe ser considerado como la guía práctica, profesional del perito en la aplicación de las ciencias forenses que permita la aplicación adecuada del formato del informe de la perica social. La aplicación y uso del mismo debe ser utilizado bajo lineamientos de ética profesional, transparencia, objetividad e imparcialidad. El debido uso del mismo facilita el uso y

${ }^{1}$ Juez Penal de Pastaza. Ecuador. Correo-e: aurelioquito@gmail.com

${ }^{2}$ Presidente de Jatun Yachay Sovilex. Abogado. Correo-e: benjhiqc@yahoo.com 
manejo del formato del informe de la pericia social y proyecta su aplicación a todas las pericias solicitadas por la autoridad competente, en el nivel nacional.

Palabras claves: Coip, aplicación, ciencias, forenses

\section{Introducción.}

1.- El delito como acontecimiento que precede a la investigación criminal: hemos de partir manifestando que el termino delito deriva del vocablo latino delicto que significa en quebranto de la ley.

En el derecho romano el término delinquere se utilizó para diferenciarlo del de crimen para designar a los delicta privata (1 ). (aquellos actos ilícitos que no dan lugar a un juicio público penal, sino a uno privado, lo que significa que sólo pueden ser intentados por la parte interesada).

Servio tulio ruiz - concibe al delito como un hecho o conducta típica - como una sanción criminal cuya ecuación representa la forma más simple del delito. considera que la conducta típica es todo acontecer humano, positivo o negativo correspondiente a una descripción legislativa (tipo) violatorio de una prohibición o mandato penal que acarrea una consecuencia jurídica consistente en una sanción criminal. refiere que esta ecuación a la que hace referencia - es decir conducta típica vs sanción criminal - sirve para llegar a otras ecuaciones más complejas en las que se encuentran inmersas o comprendidas todos los requisitos y presupuestos del ilícito penal tendientes estas a construir el andamiaje esquemático del delito - refiere que este andamiaje es el que ofrecerá un sistema conceptual que permitirá su análisis completo desentrañando de esta forma el amplio contenido de cada uno de estos elementos normativos que lo conforman (2), por su parte francisco pavon vasconcelos considera que el delito debe entenderse de distintas formas - dependiendo aquello de dos aspectos: según el tiempo y según el espacio en que sea objeto de valoración jurídica, este autor encuentra siempre su fundamento en las relaciones existentes entre el hecho como producto del quehacer humano y su valoración legislativa, sostiene su tesis respecto de que a pesar de que en un principio - en los pueblos más antiguos - si bien los hechos antisociales fueron castigados desde un punto de vista objetivo, la valoración subjetiva del hecho dañoso apareció hasta el momento en que surgieron los ordenamientos legales. indica que si bien el concepto de delito ha evolucionado a través del tiempo, nunca ha prescindido de su carácter antijurídico por lo que en el tiempo siempre se ha identificado al delito como un acto contrario a la ley - sin embargo es indiscutible que esta noción ha sido complementada por distintos elementos que a la postre se han incorporado a su concepto - sabiendo indicar que normalmente esto se refieren a la voluntad y la conciencia en la ilicitud de un hecho. (3). 
Por su parte mazger observo que la antijuricidad y tipicidad no constituyen elementos autónomos sino que la conducta típica seria la selección de los ilícitos para acomodarlos dentro del derecho penal, siendo este el modo de concretarse la antijuricidad penal. Un aporte de la teoría finalista es que a través de ella, el dolo y la culpa forman parte del concepto de acción incidiendo en la culpabilidad y entonces agrega el elemento conocido como imputabilidad. (6) Al respecto y observando esta corriendo meramente legalista hemos de analizar que el coip en su artículo 18 efectivamente recoge aquello al decir:

“....art. 18.- infracción penal.- es la conducta típica, antijurídica y culpable cuya sanción se encuentra prevista en este código..”.

“... art. 25.- tipicidad.- los tipos penales describen los elementos de las conductas penalmente relevantes..."

"... art. 29.- antijuridicidad.- para que la conducta penalmente relevante sea antijurídica deberá amenazar o lesionar, sin justa causa, un bien jurídico protegido por este código....".

Del análisis realizado y siendo cual fuera el caso se debe manifestar que indiscutiblemente los doctrinarios coinciden en que el delito consiste en toda conducta antisocial que atenta contra valores fundamentales los que se encuentran socialmente establecidos, mismos que por ser contrapuestos rompen la paz social, moral, pero que sobre todo se tornan en acciones jurídicamente reprochables siendo por esto acreedores a una sanción,

Precisamente por lo nocivo que se considera la conducta delictiva y el tipo de valores en contra de los cuales atenta, el estado se ve obligado a perseguir a los delincuentes pues su conducta rompe con esta armonía social, a través de la lesión de valores fundamentales para el individuo y la sociedad siendo estos la vida - la salud - la integridad física y mental etc.

Se ha de indicar del mismo modo que el ente encargado del proceso investigativo en materia penal es la fiscalía, organismo que previo a generar una acusación que significa el consignar ya el caso ante el juez competente, está llamado a realizar una serie de actividades investigativas que le permitan eventualmente dilucidar si es procedente o no el inicio de un proceso penal.

Al respecto el coip procesalmente regula el particular en las siguientes disposiciones:

art. 454.- principios.- el anuncio y práctica de la prueba se regirá por los siguientes principios:

1. oportunidad.- es anunciada en la etapa de evaluación y preparatoria de juicio y se practica únicamente en la audiencia de juicio, los elementos de convicción deben ser presentados en la etapa de evaluación y preparatoria de juicio, las investigaciones y pericias practicadas durante la investigación alcanzarán el valor de prueba, una vez que sean presentadas, incorporadas y valoradas en la audiencia oral de juicio. 
art. 604.- audiencia preparatoria de juicio.- para la sustanciación de la audiencia preparatoria del juicio, se seguirán además de las reglas comunes a las audiencias establecidas en este código, las siguientes:

4. concluida la intervención de los sujetos procesales, si no hay vicios de procedimiento que afecten la validez procesal, continuará la audiencia, para lo cual las partes deberán:

c) solicitar la exclusión, rechazo o inadmisibilidad de los medios de prueba, que estén encaminadas a probar hechos notorios o que por otro motivo no requieren prueba, la o el juzgador rechazará o aceptará la objeción y en este último caso declarará qué evidencias son ineficaces hasta ese momento procesal; excluirá la práctica de medios de prueba ilegales, incluyendo los que se han obtenido o practicado con violación de los requisitos formales, las normas y garantías previstas en los instrumentos internacionales de protección de derechos humanos, la constitución y este código, se ha de indicar que la función de investigación se desarrolla durante la fase de investigación mejor conocida como indagación previa momento en que la fiscalía o ministerio público interviene como autoridad y no como parte - motivo por el que a sus actos se les reconoce cierto imperio - siendo esta la razón por la que se le faculta dirigir la investigación tarea para la cual se auxilia del personal del sistema especializado integral de investigación, de medicina legal y ciencias forenses o del personal competente en materia de tránsito - a través de sus peritos - cuya finalidad tendrá es determinar si la conducta investigada es delictuosa, las circunstancias o móviles de la perpetración, la identidad del autor o partícipe y de la víctima, la existencia del daño causado, o a su vez, desestimar estos aspectos, los peritos por su parte son personas que por su conocimiento técnico en determinadas materias - sea esto en: balística, medicina legal, grafoscopia, documentologia y contabilidad, su función es ilustrar y auxiliar al representante social para conocer de mejor manera los hechos que son de su conocimiento y a tener una mejor apreciación de la verdad histórica.

Si bien se establece el particular de esta manera es menester dejar claro que bajo ningún concepto puede ni podrá desdibujarse lo que se puede justificar con el aporte pericial siendo exclusivamente el elemento materialidad conforme así lo ha establecido la corte nacional de justicia en números fallos - sabiendo para ilustración citar el contenido de dicho criterio mismo que es como sigue:

“...la corte nacional de justicia, ha analizado que prueban los testigos y que prueban los peritos en materia de tránsito, indicando al respecto lo siguiente: en relación a los delitos de accidentes de tránsito. carnelutti, establecía una diferencia entre el perito y el testigo así: "el testigo solo relata, refiere, narra hechos; en tanto que el perito expresa juicios propios de su arte, ciencia y profesión y que el juzgador desconoce. el perito y el testigo proporcionan información al juzgador pero el origen es diverso, la ciencia del perito se forma en el proceso, y, la información del testigo fuera del proceso, en el sentido de que el primero actúa para lograrla en cumplimiento de un encargo del órgano judicial y el segundo sin encargo alguno. 
la semejanza que existe entre ambos medios de prueba radica en que estos exponen a través de medio oral ante el juzgador sus conocimientos para ayudar con ello al esclarecimiento de los hechos - mientras los peritos brindan conocimientos técnicos como consecuencia de la formación profesional, no han presenciado el hecho ni tienen referencias de ello, sino que son elementales portadores de un conocimiento técnico-científico al servicio de la justicia penal. los testigos en cambio dan fe sobre acontecimientos percibidos sensorialmente....".

\section{2.- El conocimiento general:}

La palabra conocimiento proviene del latín cognoscere debiendo ser entendida como la acción y efecto de conocer - entender, advertir, saber, se ha de indicar que para que sea posible el conocer deben concurrir 3 elementos fundamentales:

1.- el sujeto que conoce

2.- el objeto de conocimiento.

3.- la imagen del objeto que se conoce.

Entonces queda claro que el sujeto se constituye en el punto de partida de este proceso pues es el ente que piensa, la imagen se determina como el nexo lógico entre el objeto y la reproducción mental del sujeto, el objeto por su parte se convierte en la realidad es decir en lo que puede ser motivo de conocimiento o sensibilidad por parte del sujeto. (7).

Los objetos de conocimiento se clasifican en ideales -reales, son reales aquellos objetos que ocupan un lugar en el espacio dada su naturaleza física - tangible por ejemplo: una silla una piedra, etc. Son ideales aquellos que no ocupan un lugar en el espacio y existen únicamente en el pensamiento por ejemplo: el amor, la justicia, el bien, el mal, etc. de la misma forma en el análisis de este punto se torna relevante el estudio de la verdad y la falsedad sabiendo indicar al respecto que estos se establecen como conceptos fundamentales de todo conocimiento pues como regla principal es indispensable entender lo que es verdadero y falso, para que el conocimiento sea verdadero implica la necesidad de que el objeto sea percibido en su totalidad - es decir de forma íntegra y absoluta, por el contrario entonces, será falso el conocimiento que presenta una imagen que contiene características distintas a las que el objeto posee, de esta forma podremos decir entonces que el conocimiento verdadero es aquel que se caracteriza por la coincidencia entre la imagen de un objeto y el objeto mismo.

El conocimiento empírico: el aquel que se adquiere mediante las vivencias a través de las experiencias sensoriales, presenta características:

Ametódico: se dice que posee dicha característica porque el conocimiento empírico lo adquiere el hombre desde que nace - son estos estímulos exteriores los que arriban a la esfera de conocimiento del sujeto de manera espontánea. por ejemplo: el hombre a través del tiempo 
sabe cuándo es temporada de lluvias o de calor por presentarse características propias de estas temporadas.

Asistemático: este tipo de conocimiento no se encuentra catalogado - sistematizado simplemente atienden a las leyes de la naturaleza.

No es explicativo: no hay explicación de por medio pues no hay necesidad de aquello - en este orden de ideas todos sabemos que en las noches sale la luna - que en la mañana sale el sol, etc.

Conocimiento teológico: la teología en si trata sobre un conjunto de creencias sin necesidad que sean confirmadas por la razón y la experiencia, este conjunto de creencias son un acto de fe, en su naturaleza misma las creencias teológicas son dogmáticas en tanto no están sometidas a prueba o confirmación alguna en esencia la teología es una doctrina sobre la esencia, la existencia y los atributos de dios.

Conocimiento científico: la ciencia en el conjunto de conocimientos objetivos ya sean estos exactos o aproximados, de las cosas, mismos que se obtienen por medio del razonamiento ordenado al aplicar los métodos de la observación y la experimentación, de su estudio se desprenden leyes, mismas que tienen validez bajo determinadas circunstancias; se elaboran hipótesis cuyo principal objetivo consiste en explicar y dar coherencia al conjunto de sucesos experimentados, se ha de manifestar que el valor de las hipótesis planteadas es susceptible de verificarse a través de la experimentación es por ello que en la actualidad a la ciencia se la concibe como un sistema coherente de conocimientos objetivos que corresponden de alguna forma a la realidad o parte de ella elaborado mediante un método racional adecuado, ha de indicarse que cada ciencia en particular se caracteriza no solo por su objeto de estudio sino también por lo que estudia de dicho objeto así como por el método utilizado las ciencias en si pueden clasificarse en puras y aplicadas:

\section{Puras:}

Se centran en el conocimiento teórico de las cosas - podemos citar entre estas a las matemáticas - química.

\section{Aplicadas:}

Buscan un aprovechamiento práctico de los conocimientos de las ciencias anteriores ej: ingeniería - agricultura.

\section{Descriptivas:}

Investigan que con las cosas, como actúan, como se relacionan, cuando, como, donde y porque ej: anatomía - geografía. 


\section{Deductivas:}

Estas se construyen racionalmente y justifican sus teoremas de forma puramente deductiva sin el recurso de la experiencia empírica ej: las matemáticas - la química.

A de concluirse manifestado que las ciencias en si se constituyen en un producto de la curiosidad del hombre - mismo que buscaba una explicación racional y objetiva del mundo y del universo es así como surgió la astronomía - la geografía. del mismo modo buscaba explicar los fenómenos naturales, incluyendo al hombre mismo, no a partir de la existencia de una divinidad y de los dogmas teológicos sino a partir de la observación y la comprensión racional de las cosas.

3.- El método científico y la criminalística: la palabra método proviene del griego métodos que significa camino o vía -. entendiéndose aquello como el modo ordenado de proceder para llegar a un resultado o un determinado fin, bajo esta perspectiva entonces se puede decir que le método científico se constituye en el conjunto de pasos que sigue una ciencia con el propósito de alcanzar conocimientos validos susceptibles de ser verificados por instrumentos confiables - aspecto este que permite a través de su aplicación que el investigador deje de lado su propia subjetividad provocando que sus conclusiones sean estrictamente el resultado de la aplicación de estos pasos. al respecto y sustentados en el pensamiento de francisco bacon, las distintas etapas en que se compone el método científico son las siguientes: observación, introducción, hipótesis, experimentación, demostración o refutación de la hipótesis, tesis.

La investigación criminalística: es el procedimiento a través del cual se pueden descubrir los acontecimientos reales conforme hubieran suscitado - esto a través de la aplicación de diversas técnicas específicas - mismas que en debida forma aplicadas permitirán alcanzar la verdad histórica de los hechos probablemente constitutivos de delito - aspecto que permitirá su posterior persecución y solución, será necesario puntualizar que en la investigación de un hecho delictivo se realiza todo un procedimiento metodológico iniciando el mismo por lo siguiente:

1. Conservación y preservación de la escena

2. Identificación, fijación y recopilación de indicios

3. Aplicación de una adecuada cadena de custodia respecto a esos indicios.

4. Estudio y análisis de los mismos

5. Completar el proceso de transformación técnico jurídica de vestigio - indicio evidencia y prueba en el proceso penal.

Sobre el uso apropiado de los métodos de investigación criminal, hay que manifestar que en derecho penal no existe una regla específica en la cual se precise la utilización de cualquier método para la obtención de la verdad - por el contrario, desde la misma constitución hasta las leyes secundarias prohíben ciertos métodos para la obtención de 
datos como la tortura - los palos - los azotes - la intimidación. de la misma forma para la realización de ciertos procedimientos se debe observar el contenido expreso de la ley como, por ejemplo - allanamientos - detención de personas, debe tenerse claro siempre que los métodos de investigación a utilizarse deben ser ideales de acuerdo al proceso en que serán utilizados pues no hacerlo tornaría muchas veces los procedimientos en inoficiosos e incluso arbitrarios, para poder definir aquello el investigador necesariamente debe contestarse a las preguntas que siguen: ¿que?, ¿quien?, ¿como?, ¿cuando?, ¿ donde?, ¿con que?, ¿por que?.

Es de indicar que el apoyo de peritos en aquello se torna fundamental a efectos de poder llegar a la búsqueda de la verdad histórica, toda vez que el conocimiento técnico de aquellos podrá dar luces en lo que respecta al avance y progreso del proceso investigativo .

\section{4.- Utilidad de la criminalística}

En el ministerio público hay quienes consideran que la representación social de la fiscalía se desprende de su carácter de institución pública, responsable de promover la defensa de aquellos que se encuentran en un claro estado de indefensión y desventaja ante terceros, se ha de indicar que al referirnos a este tema estamos dirigiendo la protección a la víctima del delito (victima directa) y la sociedad en su conjunto (victima absoluta) mismas que fruto de un acto delictivo se ven amenazadas motivo por el que desde esta perspectiva es obvio que el papel investigador de la fiscalía es crucial para el cumplimiento de sus primeros objetivos - que en este caso sería el desarrollo de una investigación efectiva.

La investigación penal efectiva, es aquella investigación que produce resultados de eficiencia, de eficacia y de justicia material, que implica la utilización de un método, de una técnica que nos permite planear la investigación, con ello establecer un orden en la actividad investigativa, debemos tener énfasis en indicar que la razón principal para utilizar una técnica radica en que la investigación no puede dejarse al azar, a lo que pueda suceder, sino que debe tener una planeación, una dirección, conducción y control que aseguren la obtención de resultados a corto plazo y permitan prever lo que podría suceder a largo plazo.

En la calificación jurídica, la complejidad de los hechos, podrán generar cierto grado de dificultad para calificarlos; es decir, encuadrarlos o enmarcarlos dentro de una o varias figuras penales - no obstante, partiendo que en nuestra investigación se maneja una calificación provisional pues la formulación de cargos no constituye acusación sino simplemente el pedido del inicio del proceso penal, es imprescindible como equipo, realizar un análisis concienzudo a fin de ser lo más acertado posible en calificarlos y aunque al final pueda variar la calificación - esto a través de figuras como la reformulación de cargos - es el abordaje o desarrollo de cada uno de los elementos del tipo penal, lo que definirá el éxito en la investigación y arribar a la conclusión correspondiente que podría incluso enviar el caso al archivo - pues conforme se sabe al concluir la etapa de instrucción - de conformidad a lo 
previsto en el art. 600 del coip el fiscal tiene total amplitud de acción a afectos de presentar o no una acusación formal.

Respecto al manejo de hipótesis en la investigación, la definición de hipótesis es una proposición de un hecho que permite llegar a demostrar otros hechos, la construcción de una hipótesis, debe estar sometida a las reglas de la lógica para evitar que este instrumento vicie las explicaciones, en conclusión, hipótesis es: una explicación tentativa que necesita de mayor información para ser confirmada o denegada.

Lo requisitos para la elaboración de hipótesis, para formular la hipótesis debe cumplirse algunos requisitos, entre ellos:

- formularse en términos claros, es decir, emplear palabras precisas que no den lugar a múltiples interpretaciones.

- tener un referente empírico (basado en la experiencia), hace que pueda ser comprobable, una hipótesis sin este referente, se transforma en un juicio de valor, que al no poder comprobarse y verificarse, carece de validez para la ciencia.

Respecto a la teoría del caso: desarrollada la investigación conforme al plan estratégico de investigación - que se plantea aplicarse en nuestro sistema judicial - y verificada la hipótesis principal que se formuló, surge la teoría del caso - esta constituye la narración sucinta de los hechos penalmente relevantes o de la historia que el fiscal va a presentar para que el juez la acepte. en términos generales esta historia se empieza a armar como un rompecabezas desde la noticia criminal y se va completando a medida que se recolectan las evidencias para comprobar o desvirtuar la hipótesis inicialmente formulada. con posterioridad habrá que ir añadiendo y desechando elementos según el desarrollo de la investigación, en el cual tiene gran importancia el plan estratégico de investigación a implementarse.

Elementos de la teoría del caso.

Fáctico: son los hechos objetivos que queremos presentar, es la historia propiamente dicha que se obtiene por medio de la noticia criminal y se va acomodando de acuerdo a los elementos probatorios e información que se vaya obteniendo. la construcción de lo fáctico se facilita si se aplica una secuencia cronológica de lo penalmente relevante que permite que todos comprendan lo sucedido y que se pueda comparar la historia con los diferentes tipos penales para encajarla en uno de ellos.

Jurídico: son los elementos de tipo normativo aplicables a lo fáctico. el fiscal debe saber qué evidencias necesita para suplir las exigencias técnico-jurídicas del tipo penal o de las normas que se vayan a utilizar en su teoría del caso. es encuadrar los hechos en el derecho. lo anterior, para que el juez o tribunal advierta claramente que se le está presentando una relación de hechos ilícitos atribuibles a una o varias personas y debidamente relacionadas entre sí (nexo causal). 
Probatorio: son los elementos probatorios recopilados hasta el momento que permiten acreditar la existencia del hecho, el delito y la responsabilidad. la evidencia debe organizarse de tal forma que se apoye en la teoría fáctica y jurídica. para efectos de la presentación de la evidencia, el equipo de trabajo debe establecer prioridades en su organización, por ejemplo el orden de presentación de testigos y peritos, de la evidencia documental, directa o circunstancial. esto según lo que sea más conveniente para probar la teoría del caso que presentará el fiscal. la teoría del caso debe ser la trama para la "obra" del fiscal, que es el juicio. como cualquier buena trama debe incluir igualmente algunos elementos típicamente necesarios como los personajes, los motivos, los escenarios, los elementos temporales y la acción, de tal manera que cualquier persona pueda comprender lo que pasó. igualmente, se debe identificar el objeto de prueba y la evidencia con la que se cuenta y con la cual se pueda probar la existencia del delito en todos sus elementos típicos, así como la responsabilidad del procesado. en este momento, adquiere nuevamente gran importancia, el plan estratégico de investigación porque permite conceptualizar todos los aspectos del caso, organizarlos y, en esta medida presentarlos en el juicio. también permitirá controlar la audiencia, las objeciones que se presenten y los debates de las partes.

Se concluye entonces que la criminalística en el proceso investigativo es determinante a efectos de poder llegar a la consecución del fin mismo de dicho proceso que es la búsqueda de la verdad histórica en cada proceso tendiente esto a garantizar el derecho de las víctimas que es en sí saber cómo efectivamente suscitaron los hechos.

\section{Metodologia.}

El presente estudio es de carácter exploratorio debido al objeto de estudio y de alcance descriptivo en relación a los objetivos planteados.

La investigación es de tipo cuantitativo debido al objeto de estudio y la metodología necesaria.

\section{Resultados.}

Sistema penal acusatorio

- Cadena de custodia: finalidad, autenticidad

- Pruebas: autenticación, libertad probatoria (aunque el método por excelencia es la cadena de custodia)

“....Esta judicatura ha tenido oportunidad de definir que la autenticación de evidencias físicas tiene un claro contenido factual, por lo que es un tema de prueba referido a la demostración de que "una evidencia, elemento, objeto o documento es lo que la parte que lo aporta dice que es". En esta medida, a efectos de llevar a cabo la autenticación de las evidencias físicas, aunque en todos los casos debería prevalecer la sujeción a los protocolos de cadena de custodia, la parte que la aporta se encuentra en libertad de solicitar los medios probatorios que considere más adecuados e idóneos para su demostración, prevaleciendo en tal sentido 
el principio de libertad probatoria que inspira el sistema procesal regulado en el art, 454 N.4 del COIP que dice: Libertad probatoria.- Todos los hechos y circunstancias pertinentes al caso, se podrán probar por cualquier medio que no sea contrario a la Constitución, los instrumentos internacionales de derechos humanos, los instrumentos internacionales ratificados por el Estado y demás normas jurídicas.

Así, la judicatura ha subrayado la obligación constitucional y legal de sujeción a la cadena de custodia como método de autenticidad por excelencia, con la que se pretende el aseguramiento de las evidencias físicas, a fin de evitar su alteración, modificación, suplantación o falseamiento, lo que determina la vigencia del principio de mismidad, según el cual, la evidencia exhibida en los estrados judiciales debe ser la misma recogida en la escena del delito o en otros lugares en el curso de las actuaciones adelantadas por los investigadores........No obstante lo anterior, también se ha precisado que si por alguna razón no se cumple con la obligación constitucional y legal de someter las evidencias físicas al procedimiento de cadena de custodia, admite que su autenticidad se pueda acreditar por cualquier medio de conocimiento, en virtud, como se ha dicho, del principio de libertad probatoria, carga demostrativa de la parte que las presente.

Por eso, tratándose de evidencias físicas que son únicas o identificables a simple vista por sus características externas, o aquellas que son susceptibles de ser marcadas y que de esa manera se hacen identificables, el protocolo de cadena de custodia puede ser suplido como procedimiento de autenticación a través de la presentación de testigos que tengan conocimiento "personal y directo" de los hechos que pondrán en conocimiento de la autoridad judicial.

En tal evento, la parte debe ofrecer los medios probatorios tendientes a la articulación de los factores que, en orden a establecer su pertinencia, determinen la mismidad de la evidencia física, esto es, presentando los testimonios a través de los cuales se pueda demostrar que el instrumento recogido es el mismo presentado como prueba ante el juez de conocimiento.

De acuerdo a la normativa penal vigente, correspondía a la Fiscalía la demostración de la autenticidad de la cuestionada evidencia física. Esa obligación material en manera alguna puede ser obviada y mucho menos sustituida con predicados presuntivos como el de la buena fe y la prudencia, cuando en realidad la existencia del arma de fuego constituye para el delito imputado el eje de la tipicidad de la conducta endilgada.

Tampoco puede el Juzgador aducir, que no se haya probado algún resquemor de los funcionarios de policía hacia el acusado como sustento de un interés en perjudicarlo, para dar por acreditada la autenticidad del medio de prueba. 
Ello significaría no solamente una inaceptable inversión de la carga de la prueba sino, más grave aún, una desviación en el curso del tema de prueba ante la exigencia absurda de acreditar por parte del procesado un interés maligno por afectar sus intereses, cuando en realidad correspondía al acusador la concreta y material demostración de cada uno de los elementos estructurales de la conducta punible.

Así las cosas, no es posible determinar que el arma de fuego al que en extenso se refirió el perito en criminalística, sea la misma recogida en el lugar de los hechos por los agentes de policía y que, según la teoría de la Fiscalía, era portada sin permiso de autoridad competente por el acusado.

Dicha imposibilidad, derivada de la ausencia de prueba sobre los hechos presentados en la acusación, impide la emisión de un juicio de reproche porque evidentemente no fue desvirtuada la presunción de inocencia del acusado en tanto se omitió la demostración de uno de los elementos que estructuran el tipo penal, esto, el arma de fuego como objeto de porte no autorizado por las autoridades correspondientes.

\section{Referencias bibliográficas.}

Real academia española - diccionario de la lengua A-G, 21 Edición Espasa - Calpe. España 1992, p. 450.

Tulio Ruiz, Servio, La estructura del delito, Editorial Temis, Colombia, 1978, pp. 5-6

Pavón Vasconcelos - imputabilidad e inimputabilidad 2da edición - editorial Porrúa México 1989 p.p 12-13.

Pavón Vasconcelos - Op cit.

Díaz de Leon Marco Antonio Diccionario de Derecho Procesal Penal - México 1989 p. 582.

Conde - Pumpido Ferreiro, Cándido Derecho Penal - Parte General Editorial Colex, España 1990 p.p 94-95.

Abbagnano Nicola Diccionario de Filosofía 13ª - Fondo de Cultura Económica 1996, pp. 216-226.

Moreno González Rafael - Manual e Introducción la criminalística Edicion Porrúa México 2006 p. 22.

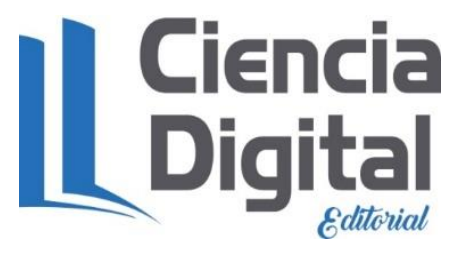


Para citar el artículo indexado.

Quito A. (2019) El coip y su relación con las ciencias forenses. Revista electrónica Ciencia Digital 3(1.1), 171-21. Recuperado desde:

http://cienciadigital.org/revistacienciadigital2/index.php/CienciaDigital/article/view/356/774

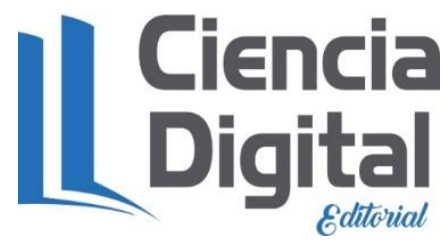

El artículo que se publica es de exclusiva responsabilidad de los autores y no necesariamente reflejan el pensamiento de la Revista Ciencia Digital.

El articulo queda en propiedad de la revista y, por tanto, su publicación parcial y/o total en otro medio tiene que ser autorizado por el director de la Revista Ciencia Digital.
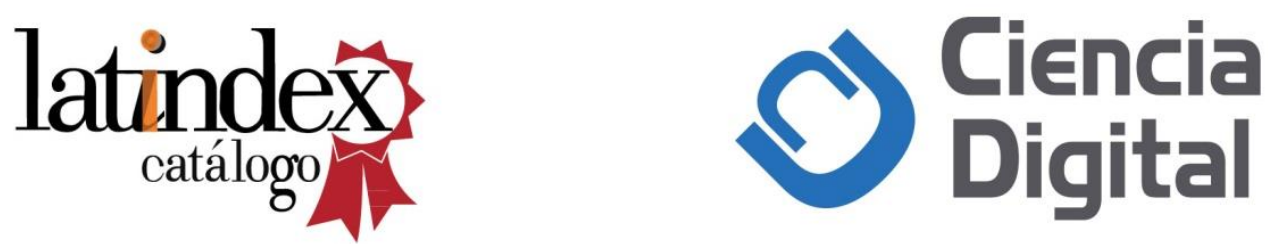